\title{
EF-hand domain containing 2 (Efhc2) is crucial for distal segmentation of pronephros in zebrafish
}

Praveen Barrodia ${ }^{1,2}$, Chinmoy Patra ${ }^{3,4}$ and Rajeeb K. Swain ${ }^{1 *}$

\begin{abstract}
Background: The blood filtering organ in zebrafish embryos is the pronephros, which consists of two functional nephrons. Segmentation of a nephron into different domains is essential for its function and is well conserved among vertebrates. Zebrafish has been extensively used as a model to understand nephron segmentation during development. Here, we have identified EF-hand domain containing 2 (Efhc2) as a novel component of genetic programme regulating nephron segmentation in zebrafish. Human EFHC2 is a protein with one predicted calcium-binding EFhand motif and three DM10 domains, whose function is unknown. EFHC2 has been implicated in several brain-related genetic diseases like Turner syndrome and juvenile myoclonic epilepsy. However, there is limited information on its normal physiological function.

Results: efhc2 mRNA is primarily expressed in the pronephros of zebrafish embryos. Other sites of expression include olfactory placode, notochord, otic vesicle, epiphysis and neuromast cells. Morpholino antisense oligonucleotidemediated knock-down of Efhc2 resulted in defects in pronephros development and function in zebrafish embryos. Efhc2 knock-down leads to expansion of distal early segment of pronephros, whereas, the corpuscle of stannius and distal late segments were reduced. The number of multi-ciliated cells (MCC) that are present in a salt-and-pepper fashion throughout the middle of each nephron and vital for fluid flow were also reduced. It is known that retinoic acid (RA) signaling regulates pronephros segmentation in vertebrates and we show that Efhc2 function is crucial for nephron segmentation in zebrafish. Our data suggests that RA and Efhc2 function independent of each other in pronephros segmentation. However, Efhc2 and RA synergistically regulate MCC development.
\end{abstract}

Conclusion: In this study, we have identified Efhc2 as a regulator of segmentation of the distal part of nephron and pronephros function during zebrafish development.

Keywords: Pronephros, Retinoic acid, Multi-ciliated cells, Efhc2, Zebrafish

\section{Background}

The vertebrate kidney is an excretory organ that arises from the intermediate mesoderm during embryogenesis [1]. The functional and structural unit of a kidney is the nephron [2]. Kidney maintains the blood plasma by filtration, reabsorption, secretion and excretion. Based on the complexity of nephron, kidneys are classified into three categories; pronephros, mesonephros

\footnotetext{
*Correspondence: rkswain.ils@gov.in

1 Institute of Life Sciences, Nalco Square, Chandrasekharpur,

Bhubaneswar, Odisha 751023, India

Full list of author information is available at the end of the article
}

and metanephros. In mammals, pronephros is vestigial, mesonephros is embryonic kidney and metanephros is adult kidney [3]. In fishes and frogs, pronephros serves as an embryonic excretory organ [4]. Zebrafish pronephros is a simple organ made up of two nephrons originating from intermediate mesoderm [5]. These two nephrons share a common glomerulus at the anterior end and are joined together by a single cloaca at the posterior end. By $24 \mathrm{hpf}$, zebrafish pronephros can be divided into eight distinct segments that have strong resemblance to nephron segments of other vertebrates including humans [6]. These segments can be visualized 
by the expression of genes in specific segments. The glomerulus (G) (expresses $w t 1 a, w t 1 b, m a f b$ ), neck (N) (expresses pax $2 a, c d h 17, N B C 1$ ), proximal convoluted tubule (PCT) (expresses slc20a1a, slc26a2, pdzk1), proximal straight tubule (PST) (expresses trpm7, slc26a2, pdzk1), distal early (DE) (expresses slc12a1, ROMK2), corpuscle of stannius (CS) (expresses stc1, sall1), distal late (DL) (expresses slc12a3, evil, ret1), and pronephric duct (PD) (expresses gata3, evi1, ret1) [7]. Kidney organogenesis in zebrafish is under the control of several transcription factors (TFs) and signaling pathways [8]. These regulatory molecules are important for specification and proper segmentation of pronephros in zebrafish. Transcription factors pax $2 a$ and pax 8 are reported to be important in specification of pronephros in vertebrates [9]. Zebrafish pronephros can be divided into two territories; proximal and distal. The development of most anterior part, the podocyte is regulated by wt1a and notch signaling components deltaC, jagged 1b, jagged $2 a$, rbpJ, and hey1 [10]. Proximal segments of the pronephros are under the control of pax $2 a$, pax 8 and jagged $2 b$ genes [10]. Transcriptional factor irx $3 b$, evi1, and pou $3 f 3 a / p o u 3 f 3 b$ are required for the development of distal segments of pronephros [6]. The $h n f 1 b a$ and $h n f 1 b b$ are required for proper nephron patterning by regulating the expression of other genes, like pax $2 a$ establishes boundary of podocyte and the neck by directly inhibiting $w t$ mediated podocyte formation [11]. Knock-down of $h n f 1 b a$ and $h n f 1 b b$ showed defects in the formation of proximal and distal segments of pronephros, indicating their role in nephron segmentation [12, 13]. Caudal type homeobox $(c d x)$ transcription factors such as $c d x 4$ and $c d x 1 a$ have been shown to regulate position of pronephros along the anterior-posterior (A-P) axis [7]. The transcription factor mecom and sim $1 a$ are required for formation of the distal tubule and restriction of proximal segments of the nephron [14].

The role of retinoic acid (RA) signaling in the proximal-distal segmentation of pronephros is well explored during zebrafish embryogenesis [7, 15]. Zebrafish embryos deficient in RA synthesis show expanded distal segments, whereas the proximal segments are either reduced or completely absent. Perturbation of retinoic acid signaling results in severe defects in nephron segmentation and pronephros function [6, 7]. Conversely, exogenous treatment of RA leads to the formation of pronephros with expanded proximal segments and reduced distal segments. These results indicate that RA promotes proximal segmentation and limits the formation of distal segments [7]. In this study, we show that Efhc2 is required for the proper segmentation of the distal parts of pronephros and development of multiciliated cells (MCC).

\section{Results}

\section{Expression pattern of efhc2 in zebrafish embryos}

Whole-mount mRNA in situ hybridization (WISH) and semi-quantitative RT-PCR were performed to study the spatio-temporal expression pattern of efhc2 during zebrafish development. Expression of efhc 2 was first observed at $6 \mathrm{hpf}$ by RT-PCR and express during the early development period (Fig. 1a). WISH data showed that efhc2 mRNA was expressed ubiquitously at $6 \mathrm{hpf}$ (Fig. 1b). By 9 hpf, its expression was localized to kupffer's vesicle (KV), a transient organ containing ciliated cells (Fig. 1c). At $12 \mathrm{hpf}$, efhc2 is expressed in the intermediate mesoderm, notochord and otic vesicle (Fig. 1d). In $24 \mathrm{hpf}$ embryos, efhc2 is expressed in pronephros, olfactory placode, notochord, otic vesicle, epiphysis, and tail bud. efhc2 is not expressed in the glomerulus and neck segments of the pronephros, but its expression starts at the PCT. More intense expression of efhc 2 was found in the proximal part of PCT compared to the distal part of the same segment (Fig. 1e, f). Strongest expression of efhc2 can be seen in the pronephric tubule segments; PST and DE. Its expression is low in DL and PD (Fig. 1e-g). Histological analysis of two colour WISH of efhc2 and $p d z k 1$ [7] confirmed the expression of efhc2 in pronephros (Fig. 1h-j). Both $36 \mathrm{hpf}$ and $48 \mathrm{hpf}$ embryos showed expression of efhc2 in the pronephros, olfactory placode, notochord, otic vesicle, epiphysis, and tailbud (Fig. 1k, l). At $72 \mathrm{hpf}$, efhc2 expression was observed in neuromast cells and olfactory placode (Fig. $1 \mathrm{~m}$ ). Expression pattern indicates the association of efhc2 with pronephros morphogenesis. Thus, we sought to address the function of efhc2 in pronephros development in zebrafish.

\section{Knock-down of Efhc2 reveals its role in pronephros development}

Zebrafish efhc 2 gene contains 16 exons. A morpholino antisense oligo targeting exon-3/intron-3 splice donor junction was designed to block the pre-mRNA splicing of efhc2 (efhc2-Mo). Corresponding mis-match morpholino antisense oligo (efhc2-MM) containing 5 mismatches compared to efhc2-Mo that was predicted not to affect normal splicing of efhc 2 pre-mRNA was used as a control. efhc2-Mo was injected in different quantities (1-4 ng) into one-cell stage embryos and its effect on RNA splicing was checked at $24 \mathrm{hpf}$ by RT-PCR using forward and reverse primers corresponding to exon-1 and exon-4 respectively. As expected, RT-PCR amplification of efhc 2 cDNA prepared from isolated RNA from $24 \mathrm{hpf}$ wild-type and efhc2-MM injected control embryos resulted in a 651 bp product, whereas the efhc2-Mo 


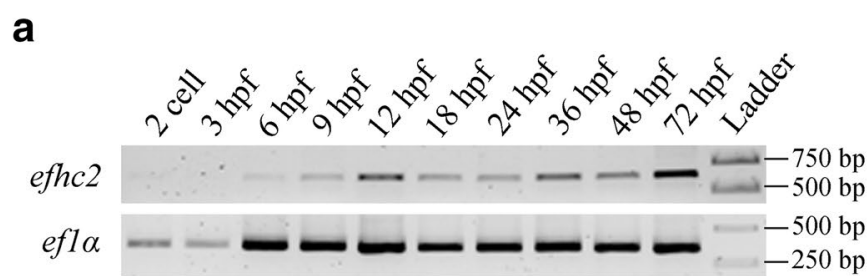

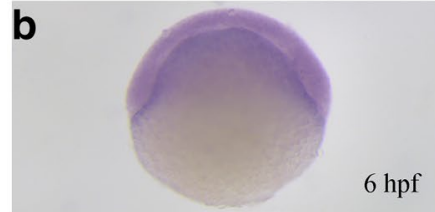

C
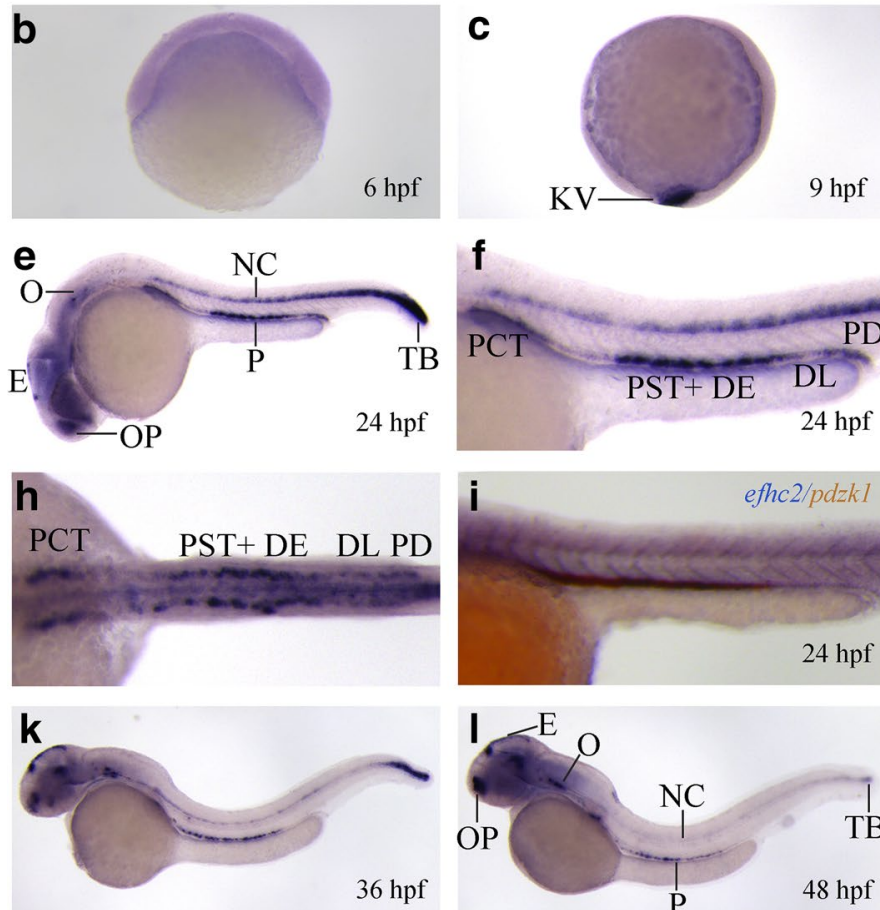

f
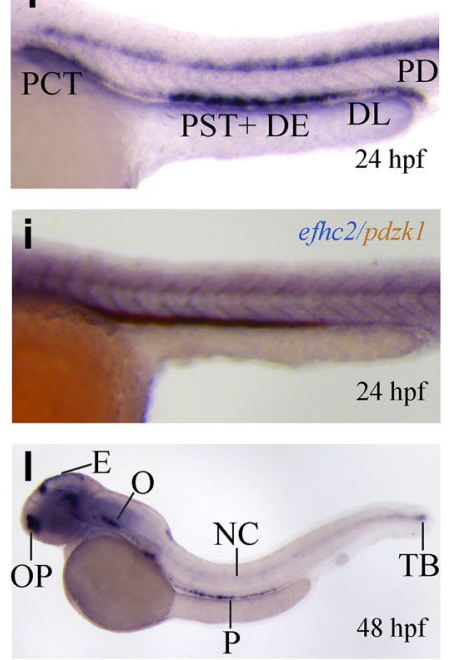

d
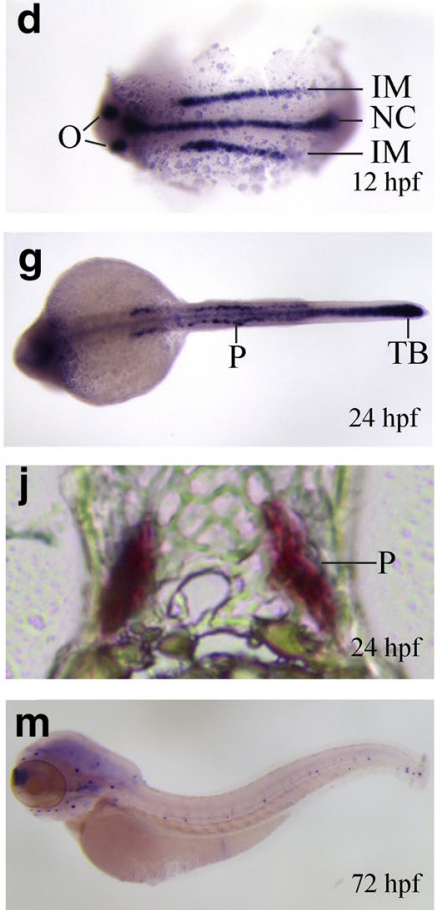

Fig. 1 Expression of efhc2 mRNA during zebrafish development. RT-PCR and whole embryo mRNA in situ hybridization (WISH) was carried out on different stages of zebrafish development. a RT-PCR analysis of efhc2 mRNA expression during zebrafish development. efla was used as loading control. b Ubiquitous expression of efhc2 at 6 hpf. c efhc2 expression in kupffer's vesicle (KV) at 9 hpf. $\mathbf{d}$ Expression in the intermediate mesoderm (IM), notochord (NC) and otic vesicle (O) at 12 hpf. e Expression of efhc2 in olfactory placode (OP), pronephros (P), epiphysis (E), notochord (NC), otic vesicle (O) and in tailbud (TB) at $24 \mathrm{hpf}$. $\mathbf{f}$ Magnified image of zebrafish trunk show strong expression of efhc2 in PST and DE and low expression in PCT, DL and PD. $\mathbf{g}$ Dorsal view of 24 hpf embryos showing its expression in pronephros. $\mathbf{h}$, i Two colour WISH of pdzk 1 and efhc2. J Section through trunk showing efhc2 expression in pronephros (P). k, I Expression of efhc2 in epiphysis, pronephros, notochord, otic vesicle and in tail bud at 36 and $48 \mathrm{hpf}$. (M) Expression of efhc2 in neuromast cells and olfactory placode at 72 hpf

injected embryos showed a 311 bp product (Fig. 2a). Both $651 \mathrm{bp}$ and $311 \mathrm{bp}$ fragments were cloned into pCR-BluntII-Topo vector and sequenced. The sequencing data confirmed that injection of efhc2-Mo results in deletion of a $340 \mathrm{bp}$ fragment containing both Exon-2 and 3 (Additional file 1: Figure S1). This indicates that injection of efhc2-Mo leads to mis-splicing of efhc2 mRNA whereas injection of efhc2-MM has no effect on normal splicing. Based on above observations, 2 ng of efhc2-Mo was injected into embryos for further characterization of its loss-of-function. Same quantity of efhc2-MM was used as control. The un-injected or efhc2-MM injected embryos did not show any morphological defects (Fig. 2b). The efhc2-Mo injected embryos, however, exhibited phenotypic defects such as slightly curved body, mild pericardial oedema and hydrocephalus typically observed in embryos where pronephros development and function is impaired (Fig. 2b). Similar morphological defects were observed when an independent morpholino antisense oligo designed to block efhc2 translation (efhc2-ATGMo) was injected (Additional file 2: Figure S2A).

\section{Knock-down of Efhc2 results in impaired pronephros function}

Next, we asked if knock-down of Efhc2 affects the function of the nephrons. Functional zebrafish nephrons of pronephros can easily clear $40 \mathrm{kDa}$ dextran injected into the cardinal vein [16]. The efhc2-Mo 


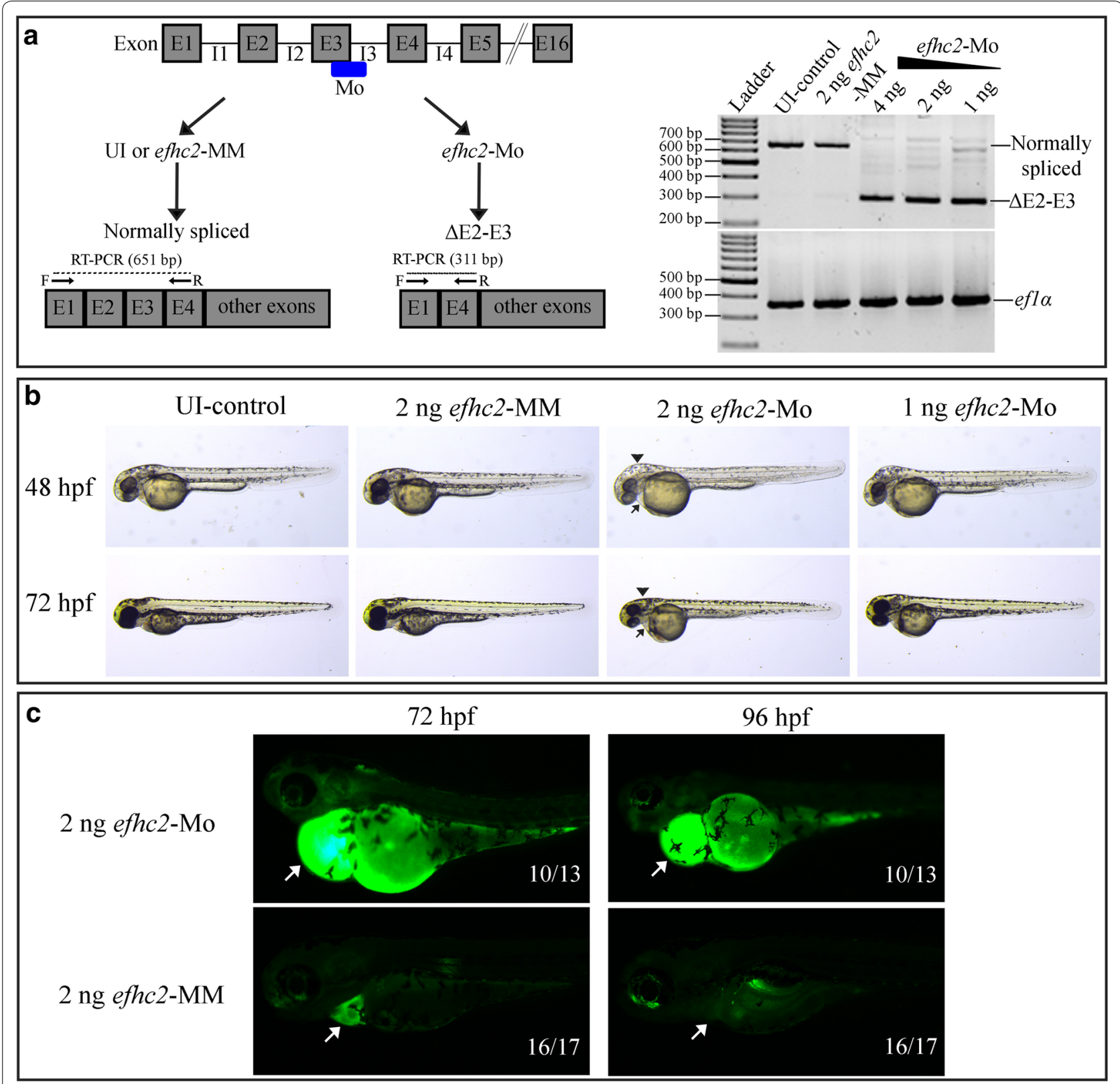

Fig. 2 Effect of Efhc2 knock-down on pronephros development and function. a Schematic representation of the binding of the splice-blocking efhc2 morpholino (efhc2-MO) targeting exon-3 and the forward and reverse primers used in RT-PCR to verify its effect. RT-PCR on RNA extracted from $24 \mathrm{hpf}$ uninjected embryos or embryos injected with efhc2-MM control showed a 651 bp product as expected. The embryos injected with efhc2-MO showed a 311 bp fragment confirming the blocking of normal splicing of efhc2 by this morpholino. $\mathbf{b}$ efhc2-Mo morphants showed morpholino dose-dependent developmental defects as compared with wild-type and mismatch controls. Arrow indicates mild pericardial oedema and arrowhead indicates hydrocephalus. c Fluorescent $40 \mathrm{kDa}$ dextran was injected into cardinal vain of control and efhc2-Mo morphants at $48 \mathrm{hpf}$. Accumulation of dextran in yolk and oedema shows defects in pronephros function in efhc2-Mo morphants at 72 and 96 hpf

and efhc2-MM morphants were injected with fluorescein isothiocyanate $40 \mathrm{kDa}$ dextran at $48 \mathrm{hpf}$ and the embryos were visualized at 72 and $96 \mathrm{hpf}$. efhc2-MM injected embryos were able to clear it and the dextran was not visible at 72 or 96 hpf. However, abundant fluorescent dextran was seen in embryos where Efhc2 was knocked-down (Fig. 2c). Accumulation of $40 \mathrm{kDa}$ dextran also led to severe pericardial oedema in these embryos (Fig. 2c). These data indicate that Efhc2 is crucial for pronephros function. 


\section{Efhc2 knock-down affects segmentation of distal part of the pronephros}

The nephrons are divided into different segments that reflect their function and are highly conserved among vertebrates [6, 10]. Eight distinct segments can be seen in a nephron of a zebrafish embryo [7]. We asked if lossof-Efhc2 leads to aberrant segmentation of the nephrons. Sodium-dependent phosphate transporter slc20a1a is expressed in the pronephros aligning with 5th to 8th somite at $24 \mathrm{hpf}$ and from 3rd to 7 th somite at $48 \mathrm{hpf}$ demarcating the PCT. Transient receptor potential cation channel gene trpm 7 is expressed in part of the nephron adjacent to 9th to 11 th somite at $24 \mathrm{hpf}$ and from 8 th to 11 th somite demarcating the PST segment of the pronephros. The expression domains of slc20a1a and trpm7 were identical in both efhc2-Mo and efhc2-MM injected embryos (Fig. 3a and Additional file 3: Figure S3A). This indicates that knock-down of Efhc2 does not influence segmentation of the proximal part of the pronephric tubule consisting of PCT and PST. The expression of sodium/potassium/chloride transporter slc12a1 in the pronephros next to 12 th and 13th somite at $24 \mathrm{hpf}$ and 12 th to 14 th somite in $48 \mathrm{hpf}$ demarcates the DE segment of the nephron. This expression domain of slc12a1 was not changed in efhc2-MM injected embryos. However, Efhc2 knock-down led to the expression of slc12a1 in 12 th to 15 th somite in both 24 and $48 \mathrm{hpf}$ embryos (Fig. 3b and Additional file 3: Figure S3A). This expression domain of slc12a1 in Efhc2 knock-down embryos indicates that loss-of-Efhc2 function results in the expansion of DE distally. The DE expansion was observed in $53 \%(51 / 97)$ at $24 \mathrm{hpf}$ and $69 \%(49 / 71)$ at $48 \mathrm{hpf}$ in Efhc2 knock-down embryos (Fig. 3b and Additional file 3: Figure S3A). A second morpholino designed to block efhc2 translation (efhc2-ATG-Mo) also lead to expansion in DE expression domain by 3 somites at $48 \mathrm{hpf}$ in 59\% (16/27) embryos as marked by slc12a1 expression (Additional file 2: Figure S2B).

The efhc2-Mo morphants showed significantly reduced DL (Fig. 3b and Additional file 3: Figure S3A). The expression of DL marker sodium/chloride transporter slc12a3 was confined to pronephros adjacent to 16 th and 17 th somite in $24 \mathrm{hpf}$ and $48 \mathrm{hpf}$ embryos injected with efhc2Mo. Whereas, the position of DL segment was normal in efhc2-MM injected embryos, where it was expressed next to 14 th to 17 th somite in $24 \mathrm{hpf}$ and 15 th to 17 th somite in $48 \mathrm{hpf}$ embryos (Fig. $3 \mathrm{~b}$ and Additional file 3: Figure $\mathrm{S} 3 \mathrm{~A})$. The DL reduction in efhc2-Mo morphants was $53 \%$ $(54 / 102)$ at $24 \mathrm{hpf}$ and $55 \%(42 / 77)$ at $48 \mathrm{hpf}$ (Fig. $3 \mathrm{~b}$ and Additional file 3: Figure S3A). This result was confirmed by injection of efhc2-ATG-Mo, which resulted in reduction of DE domain in morpholino injected embryos. The slc12a3 expression domain was reduced to 16 th and 17 th somite at $48 \mathrm{hpf}$ in 59\% (16/27) embryos as compared to normal expression from 15 th to 17 th somite (Additional file 2: Figure S2B). Stanniocalcin (stc1) is expressed in CS (next to somite number 15) and is involved in calcium and phosphate homeostasis. The expression level and position of stc1 was affected by knock-down of Efhc2. stc1 expression was reduced in efhc2-Mo injected embryos as compared to embryos injected with control efhc2-MM morpholino. Its localization was shifted distally next to 16 th somite, whereas efhc2-MM injected embryos showed normal expression of stc1 adjacent to 15th somite. The CS reduction in efhc2-Mo morphants was 50\% (43/85) (Fig. $3 \mathrm{~b}$ and Additional file 3: Figure S3A). efhc2-ATG-Mo injected embryos also showed the same results as seen with efhc2-Mo injected embryos. The expression level of stc1 was reduced and the expression domain was sifted from 15 th somite to 16 th somite in efhc2-ATG-Mo injected embryos (Additional file 2: Figure S2B). To confirm the specificity of phenotype caused by morpholino-mediated knock-down of Efhc2, we co-injected zebrafish efhc2 mRNA with efhc2-Mo. To check overexpression phenotype, we injected 100-300 pg of efhc2 mRNA/embryo. Embryos injected with 100200 pg mRNA develop normally. However, more than 200 pg mRNA injection leads to severe developmental defects (Additional file 4: Figure S4). Hence, we have injected $200 \mathrm{pg}$ efhc $2 \mathrm{mRNA}$ for rescue experiments. Coinjection of zebrafish efhc 2 mRNA partially rescued the effect of efhc2-Mo mediated knock-down of endogenous Efhc2. We found that the DE segment defect was rescued by $62.5 \%(20 / 32)$ at $24 \mathrm{hpf}$ and $56 \%(24 / 43)$ at $48 \mathrm{hpf}$. DL segment defect was rescued by $62.07(18 / 29)$ at $24 \mathrm{hpf}$ and $57 \%(24 / 42)$ embryos at $48 \mathrm{hpf}$. More than $92.5 \%$ of efhc2-Mo morphants were rescued for CS segment development (Fig. 3b, c). Thus, our data suggests that the pronephros segmentation phenotype is Efhc2 knock-down specific.

Taken together, our experiments suggest that lossof-Efhc2 function leads to expansion of DE distally and reduction of CS and DL segments of the distal pronephric tubule in zebrafish embryos (Fig. 3, Additional file 2: Figure S2 and Additional file 3: Figure S3). Hence, Efhc2 is essential for normal segmentation of the distal part of the pronephros.

\section{Efhc2 has no influence on Retinoic acid mediated segmentation of zebrafish pronephros}

Our findings reveal an important role for Efhc2 in the segmentation of distal part of zebrafish pronephros. Several reports suggest that RA signaling is important for nephron segmentation in vertebrates [4, 7]. RA signaling is required for the development of PCT and PST and is thought to inhibit the formation of distal segments such 

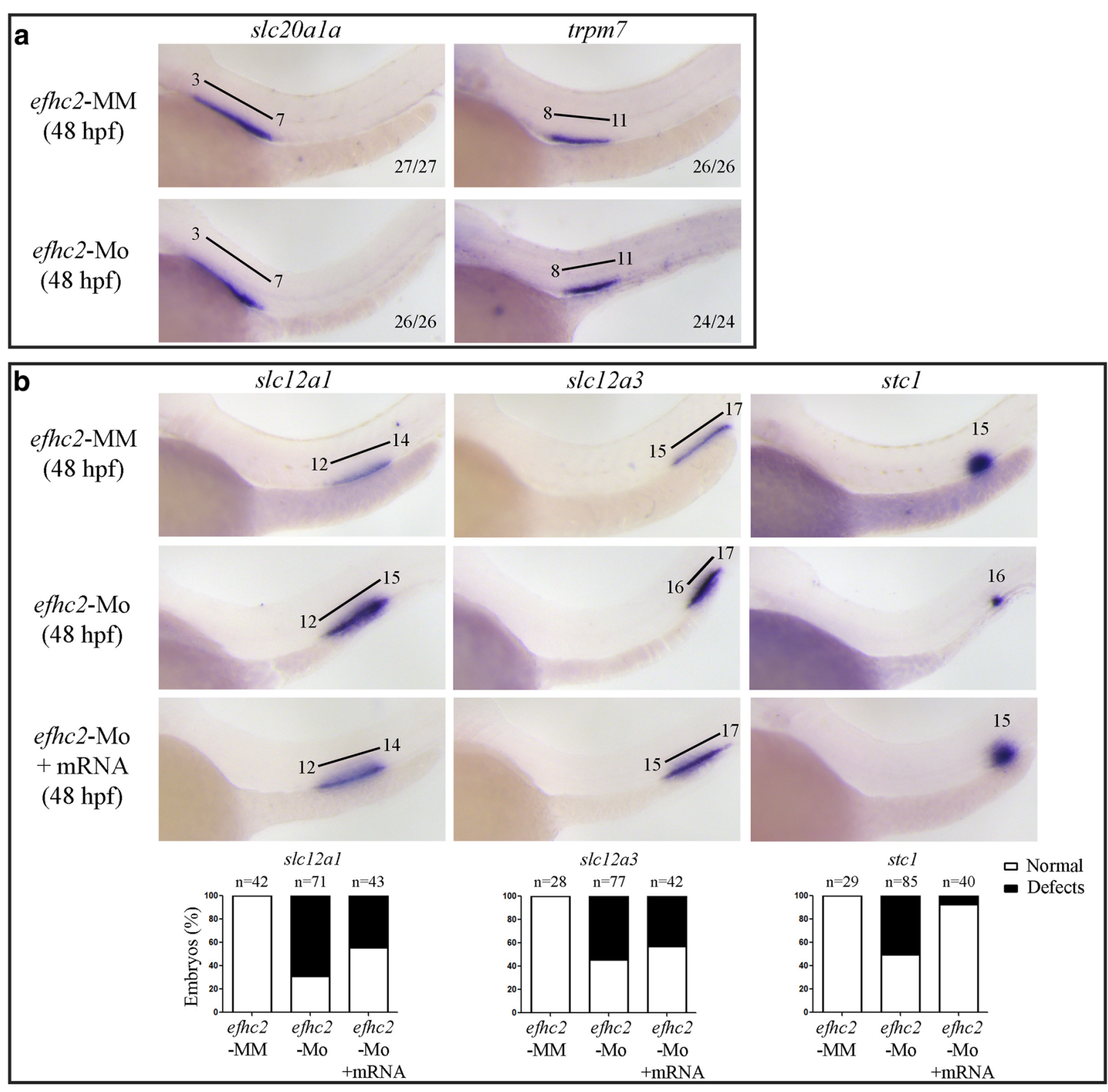

\begin{tabular}{|c|c|c|c|c|c|c|c|c|c|c|c|c|c|c|c|c|}
\hline \multirow{3}{*}{$\begin{array}{l}\text { C } \\
\text { Summary } \\
\text { (48 hpf) }\end{array}$} & Somites & 3 & 4 & 5 & 6 & 7 & 8 & 9 & 10 & 11 & 12 & 13 & 14 & 15 & $16 \mid 17$ & 18 \\
\hline & efhc2-MM & \multicolumn{9}{|c|}{ proximal segments } & \multicolumn{3}{|c|}{$\mathrm{DE}$} & \multicolumn{2}{|r|}{ DL } & \\
\hline & efhc2-Mo & \multicolumn{9}{|c|}{ proximal segments } & \multicolumn{4}{|c|}{$\mathrm{DE}$} & DL & \\
\hline & efhc2-Mo+mRNA & \multicolumn{9}{|c|}{ proximal segments } & \multicolumn{3}{|c|}{$\mathrm{DE}$} & \multicolumn{2}{|r|}{ DL } & \\
\hline
\end{tabular}

Fig. 3 Characterizing the phenotype caused by Efhc2 knock-down. a and b WISH for pronephros segment specific genes on 48 hpf morpholino injected embryos. Expression of slc20ala (PCT), trpm7 (PST), slc12al (DE), s/c12a3 (DL) and stc1 (CS) in efhc2 mismatch control (efhc2-MM) and splice-blocking morpholino (efhc2-Mo) injected embryos. The phenotype caused by Efhc2 knock-down was rescued by co-injecting efhc2 mRNA along with efhc2 morpholino. c Schematic representation of defects caused by Efhc2 knock-down 
as DE and DL in zebrafish [15]. Exogenous treatment of RA results in a pronephros with expanded PCT and PST. The DE and DL segments are either reduced or shifted distally dependent on the time and concentration of RA treatment [7]. Conversely, inhibition of RA synthesis by $\mathrm{DEAB}$ (4-diethylaminobenzaldehyde) results in reduced proximal segments and expanded distal segments [7]. Hence, we asked if Efhc2 plays any role in RA mediated inhibition of distal pronephric segment formation. The uninjected control or efhc2-Mo injected embryos were treated with exogenous RA $\left(1 \times 10^{-7} \mathrm{M}\right)$ from 9 to $16 \mathrm{hpf}$ and the formation of distal pronephric tubule segments were checked at 24 and $48 \mathrm{hpf}$. As reported by other groups, RA treated wild-type embryos had the DE segment shifted distally compared to vehicle (DMSO) treated embryos. The RA treated embryos expressed DE marker slc12a1 in a domain next to 14th to 16th somite compared the DMSO treated controls that showed normal expression domain next to 12th to 14th somite. Injection of efhc2-Mo did not shift the DE segment and the anterior domain of slc12a1 was still localized to 12 th somite. These embryos, however, had a slightly expanded DE segment. The DE segment shifted distally and expanded when efhc2-Mo injected embryos were treated with RA (Fig. 4a and Additional file 5: Figure S5). Essentially, RA treatment of Efhc2 knock-down embryos exhibited a phenotype that was a combination of both RA treatment and Efhc2 knock-down (Fig. 4a, b).

Next, we examined the effect of RA treatment on DL and CS segments in embryos lacking Efhc2. DMSO treated wild-type embryos showed expression of DL marker slc12a3 next to 15 th to 17 th somite, which was shifted distally and reduced to an expression domain next to 17th and 18th somite when treated with RA. The efhc2-Mo injected embryos had the slc12a3 expression domain reduced in nephron next to 16th and 17th somite. However, when these Efhc2 knock-down embryos were subjected to RA treatment, the DL segment was dramatically reduced to only one somite length and occupied the distal most part of the pronephric tubule next to 18th somite (Fig. 4a and Additional file 5: Figure S5). RA treatment of embryos lacking Efhc2 had a similar effect on the CS. The CS segment marker stc1, which is normally expressed next to 15th somite, was reduced and shifted distally to 18 th somite in the RA treated and 16th somite in efhc2-Mo injected embryos. This expression of $s t c 1$ was completely abolished in more than $90 \%$ embryos that lacked Efhc2 and were treated with RA (Fig. 4a).

In summary, efhc2-Mo injected embryos show two distinct and opposing effects in response to exogenous RA treatment within the distal pronephric tubule segments. RA treatment of embryos lacking Efhc2 function expands the DE segment whereas, under same conditions, the
$\mathrm{CS}$ and DL segments are much reduced (Fig. 4b and Additional file 5: Figure S5). However, the effect of RA treatment of Efhc2 knock-down embryos reflects a combination of the effects of Efhc2 knock-down and exogenous RA treatment. Hence, the effect of RA and Efhc2 on pronephros segmentation may be independent of each other.

Next, we checked the effect of lack of RA signaling on Efhc 2 mediated segmentation of distal part of the nephron. RA synthesis was inhibited by treatment of embryos with 4-diethylaminobenzaldehyde (DEAB) $\left(1 \times 10^{-5} \mathrm{M}\right)$ or 3,7-dimethyl-2,6-octadienal (citral) [17] $\left(2.5 \times 10^{-5} \mathrm{M}\right)$ from 9 to $16 \mathrm{hpf}$, and the effect of this treatment on pronephros segmentation was monitored at 24 and $48 \mathrm{hpf}$ using segment-specific marker gene expression. DEAB treatment resulted in nephrons that had the DE domain shifted proximally and the DE marker slc12a1 was expressed in a region next to 9th to 12th somite as compared to its expression next to 12th to 14th somite in vehicle (DMSO) treated embryos (Fig. 5a). However, DEAB treatment of Efhc2 knock-down embryos resulted in slc12a1 expression in nephron next to 8th to 13th somite (Fig. 5a and Additional file 5: Figure S5). Blocking RA synthesis by citral also resulted in similar changes in DE marker expression. Citral treatment of Efhc2 knock-down embryos resulted in slc12a1 expression in nephron next to 8th to 14th somite (Fig. 6a). This indicates that inhibition of RA synthesis by DEAB or citral in combination with Efhc2 knock-down leads to embryos with expanded DE segment as seen in efhc2-Mo morphants and a slight shift of this domain to proximal part of the embryos as seen in DEAB or citral treated embryos (Figs. 5b and 6b).

Inhibition of RA signaling by DEAB or citral results in expansion and slight proximal shifting, and Efhc2 knockdown results in reduced DL segment. The DL marker slc12a3 was expressed next to 13 th to 17 th somite in $\mathrm{DEAB}$ or citral treated embryos as compared to its normal expression domain of 15 th to 17 th somite in vehicle treated embryos. slc12a3 was expressed in 16th to 17th somite in Efhc2 knock-down embryos (Figs. 5a, 6a and Additional file 5: Figure S5). DEAB treatment of Efhc2 knock-down embryos resulted in slc12a3 expression in 14th to 17th somite. Citral treatment of Efhc2 knockdown embryos resulted in $s l c 12 a 3$ expression in nephron next to 15 th to 17 th somite. This indicates that DEAB or citral treatment of Efhc2 knock-down embryos had resulted in DL segment that had shifted proximally compared to both wild type or Efhc2 knock-down embryos. The DL segment in these embryos was expanded when compared to vehicle treated or Efhc2 knock-down, but there was a reduction in DL domain when compared to DEAB or citral treatment alone. These effects of RA 


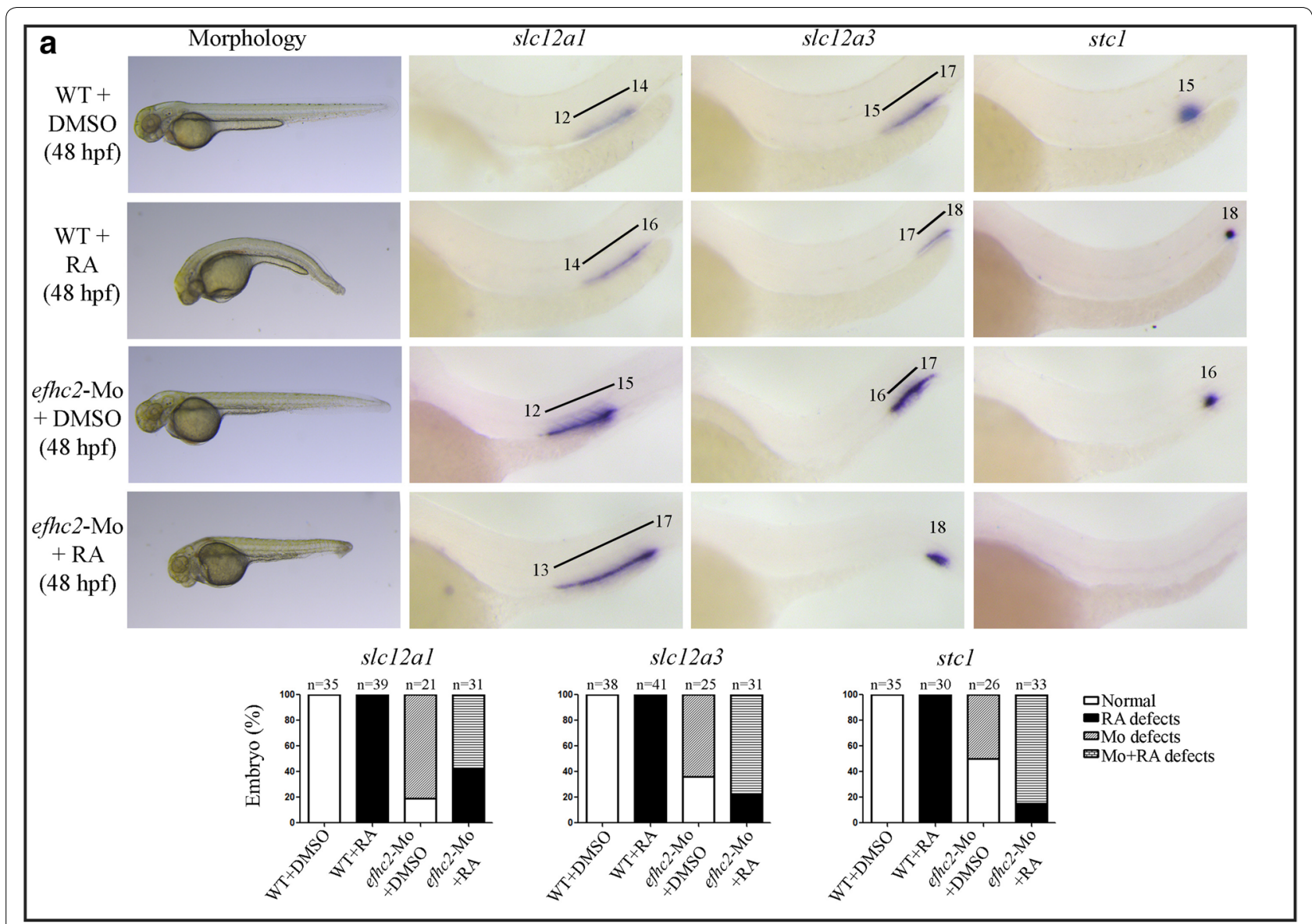

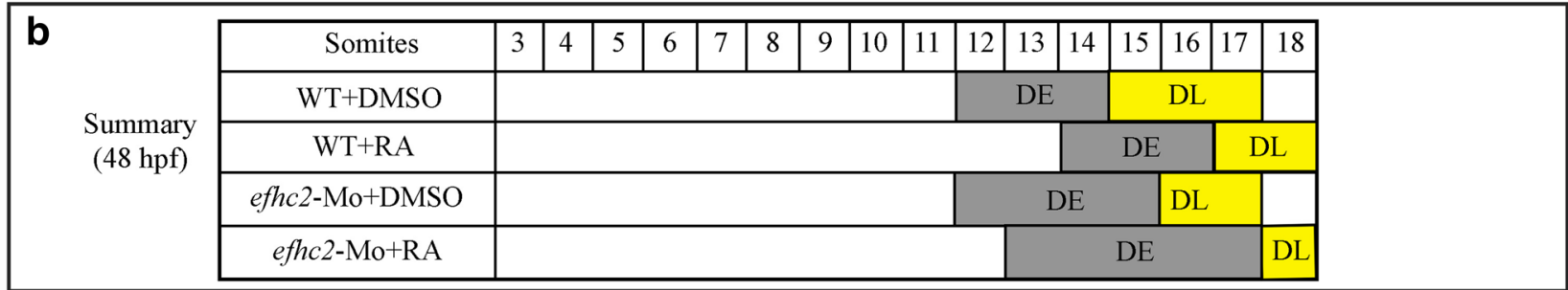

Fig. 4 Role of Efhc2 in RA mediated segmentation of pronephros development. a Wild-type embryos or efhc2 morphants were treated with RA. Morphological changes caused by RA treatment in wild-type and efhc2 morphants. WISH showing expression of s/c12al (DE), s/c12a3 (DL) and stc1 (CS). Wild-type embryos or efhc2 morphants were treated with RA $\left(1 \times 10^{-7} \mathrm{M}\right)$. efhc2 morphants treated with RA show expansion of expression domain of DE marker s/C12a1 and almost or complete loss of DL and CS markers s/c12a3 and stcl as compared with wild-type embryos treated with RA. b Summary of the effects of RA on WT and efhc2 morphants at 48 hpf

signaling inhibition on DE and DL in Efhc2 knock-down embryos is opposite of the effects seen upon exogenous RA treatment. These data support the observation that exogenous RA treatment or inhibition of RA synthesis is able to exhibit their effects in absence of Efhc2.

The CS segment present next to 15th somite was shifted proximally and expanded upon DEAB treatment. Expression of CS marker stc1 was much reduced and was shifted distally to 16th somite after Efhc2 knock-down. DEAB treatment of embryos lacking Efhc2 resulted in enhanced stc1 expression in somite 10th to 11th (Fig. 5a and Additional file 5: Figure S5). Citral treatment of Efhc2 knock-down embryos also resulted in enhanced expression of stc1 in nephron next to 10th to 11th somite (Fig. 6). This effect of DEAB or citral on efhc2-Mo morphants is exact opposite of RA treatment of same embryos where stc 1 expression was completely abolished. Taken together, these results indicate that RA signaling is able to control the formation of CS in absence of Efhc2. Hence, although both RA and Efhc2 affect 


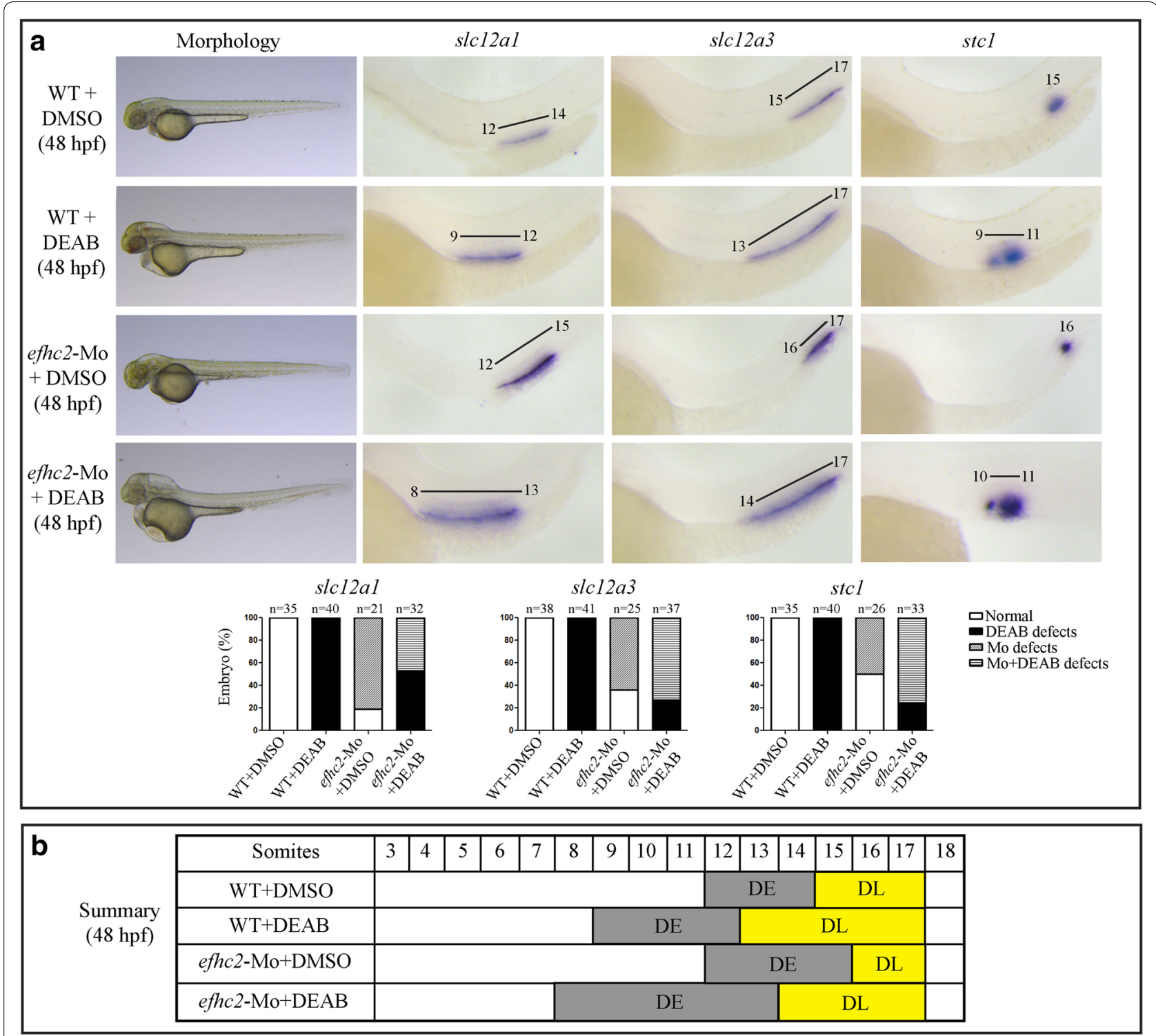

Fig. 5 Effect of blocking RA synthesis by DEAB in efhc2-Mo morphants during pronephros segmentation. a Wild-type embryos or efhc2-Mo morphants were treated with DEAB $\left(1 \times 10^{-5} \mathrm{M}\right)$. Morphological changes caused by DEAB treatment in wild-type and efhc2 morphants. efhc2-Mo morphants treated with DEAB show expansion of expression domain of DE marker s/c12a1 and slight reduction of DL and CS markers s/c12a3 and stcl as compared with wild-type embryos treated with DEAB. b Summary of the effects of DEAB on WT and efhc2-Mo morphants at 48 hpf

distal segmentation of pronephros, their functions are not interdependent.

\section{Efhc2 is required for formation of multi-ciliated cells (MCC)}

Multi-ciliated cells (MCC) are present along the PCT, PST, DE and anterior part of DL segments of pronephros in zebrafish embryos [16]. These cells can be identified by WISH using ciliogenesis genes such as odf 3 and $r f x 2$ [18]. We asked if knock-down of Efhc2 had any effect on MCC formation. The control efhc2-MM injected embryos expressed odf3 corresponding to 2 nd to 15 th somite, whereas the efhc2-Mo morphants had odf3 expression next to 8 th to 15 th somite (Fig. 7a). This suggests that embryos injected with efhc2-Mo had a muchreduced domain of $o d f 3$ expression compared to embryos injected with mis-match control. The MCC reduction in efhc2-Mo morphants was 59\% (63/106) at $24 \mathrm{hpf}$ and $61 \%(49 / 80)$ at $48 \mathrm{hpf}$ (Fig. 7a and Additional file 3: Figure S3). This effect of Efhc2 knock-down was rescued by co-injection of efhc 2 mRNA in $68.3 \%(26 / 33)$ embryos at $48 \mathrm{hpf}$ (Fig. 7a). Injection of efhc2-ATG-Mo also lead to reduction in MCC formation. Fifty five percent embryos 


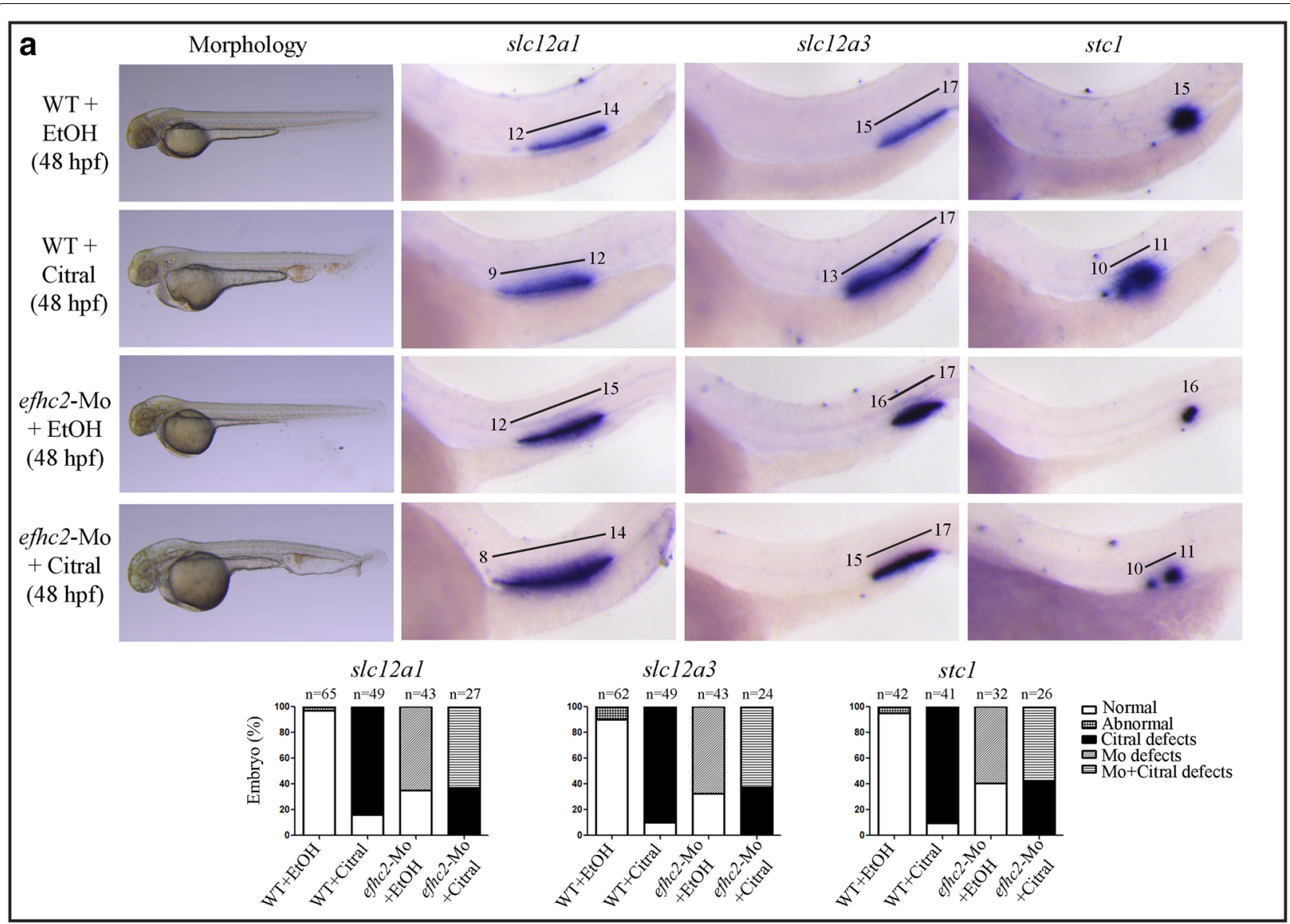

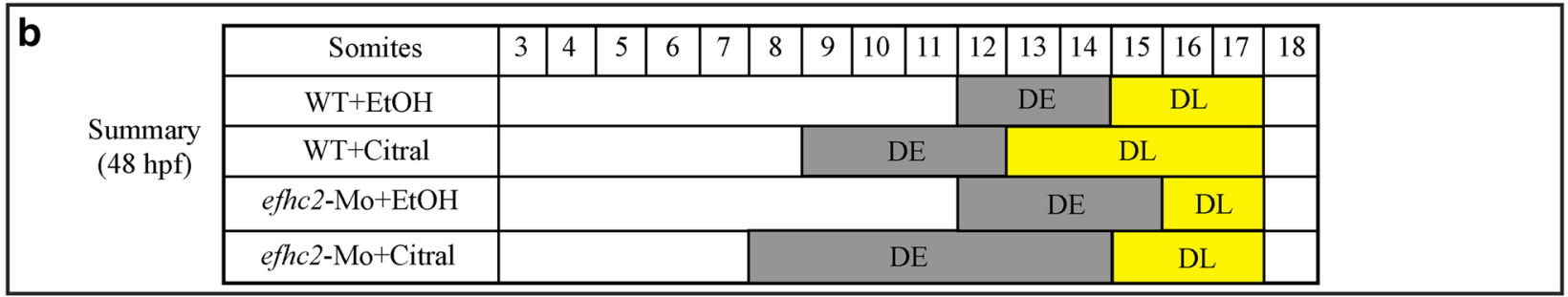

Fig. 6 Effect of RA synthesis blocker citral on pronephros segmentation in efhc2-knocked-down embryos. a Wild-type embryos or efhc2-Mo morphants were treated with citral $\left(2.5 \times 10^{-5} \mathrm{M}\right)$. Morphological defects caused by citral treatment in wild-type and efhc2 morphants. efhc2-Mo morphants treated with citral show expansion of expression domain of DE marker s/C12a1 and slight reduction of DL and CS markers s/c12a3 and stcl as compared with wild-type embryos treated with citral. b Summary of the effects of citral on WT and efhc2-Mo morphants at 48 hpf

(See figure on next page.)

Fig. 7 Role of Efhc2 and RA in MCC development. a WISH for MCC specific marker odf3 in efhc2 mismatch control (efhc2-MM) and splice-blocking morpholino (efhc2-Mo) injected embryos. The phenotype caused by Efhc2 knock-down was rescued by efhc2 mRNA. b Wild-type embryos or efhc2-Mo morphants were treated with RA. WISH showing expression of odf3 gene. efhc2-Mo morphants treated with RA show reduction of expression domain of MCC as compared to wild-type embryos treated with RA. c Wild-type embryos or efhc2-Mo morphants were treated with DEAB. WISH showing expression of odf3 gene. efhc2-Mo morphants treated with DEAB show reduction of expression domain of MCC as compared to wild-type embryos treated with DEAB. $\mathbf{d}$ Wild-type embryos or efhc2-Mo morphants were treated with citral. WISH showing expression of odf3 gene. efhc2-Mo morphants treated with citral show reduction of expression domain of MCC as compared to wild-type embryos treated with citral 

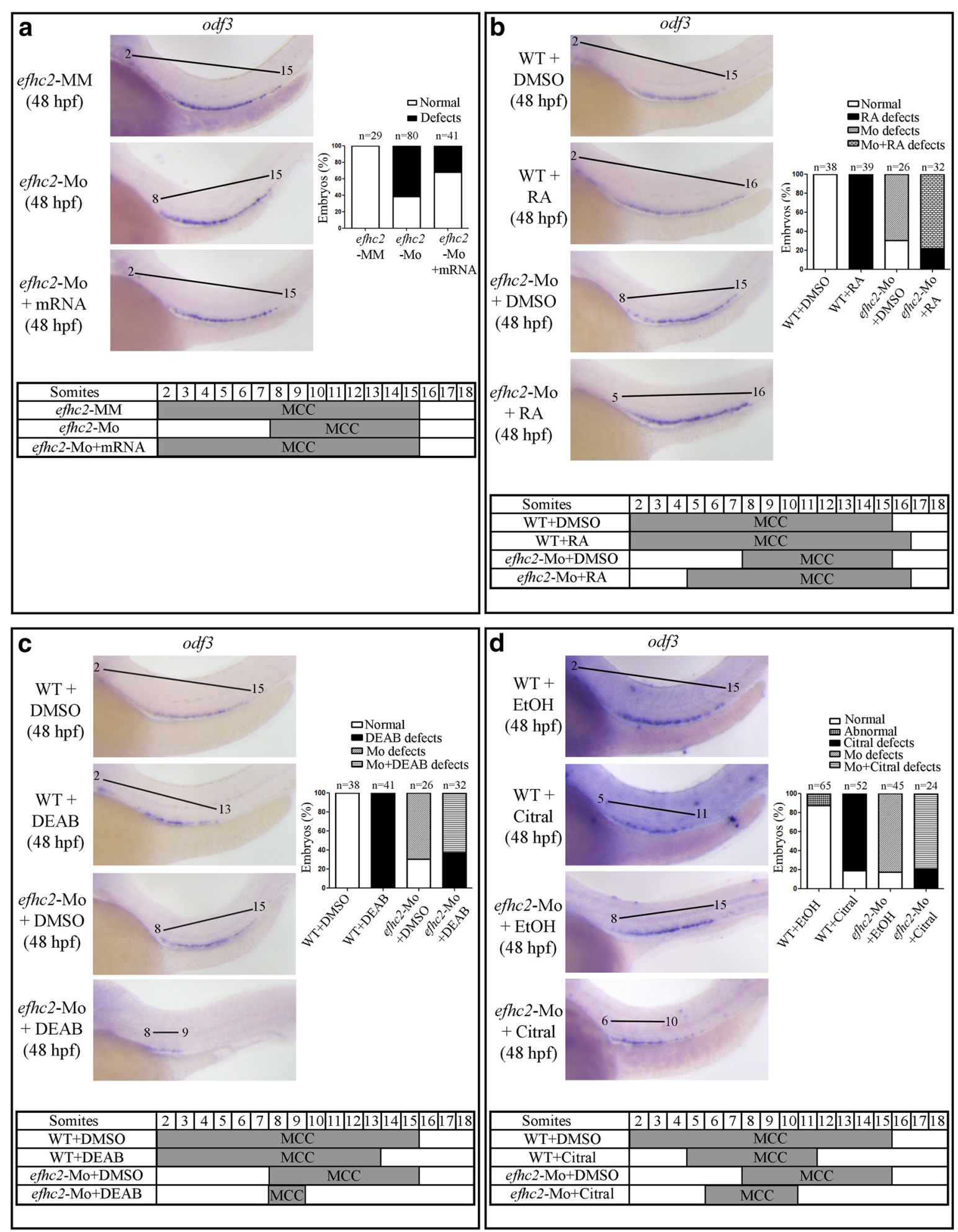
(21/38) had the odf3 expression level and domain reduced (Additional file 2: Figure S2). These observations indicate that Efhc2 positively regulates pronephric multi-ciliated cell development (Fig. 7a and Additional file 2: Figure S2).

It is known that RA signaling promotes MCC formation [16]. Exogenous treatment of RA enhances MCC number and these cells are expressed slightly distally in response to RA treatment. Conversely, treatment of embryos with RA inhibitor DEAB reduced MCC number. Knock-down of Efhc2 resulted in reduced MCC number and the expression domain of MCC marker odf3 was also reduced. Hence, we asked if Efhc2 plays any role in RA mediated MCC formation. The wild-type and Efhc2 knock-down embryos were treated with RA $\left(1 \times 10^{-7} \mathrm{M}\right)$ or RA inhibitors DEAB $\left(1 \times 10^{-5} \mathrm{M}\right)$ or citral $\left(2.5 \times 10^{-5} \mathrm{M}\right)$ from 9 to $16 \mathrm{hpf}$ and development of MCC in these embryos were examined using the expression of MCC marker odf3 at $48 \mathrm{hpf}$. The RA treated wild-type embryos showed an increase in MCC number and expansion odf3 expression domain as compared to DMSO treated embryos (Fig. 7b). The expression of odf 3 is seen in pronephros adjacent to 2nd to 16th somite in 48 hpf RA treated embryos. However, efhc2-Mo injected embryos treated with same concentration of RA showed expression of odf3 next to 5th to 16th somite in $48 \mathrm{hpf}$ embryos as compared to expression of odf 3 from 8th to 15th somite in Efhc2 knock-down alone (Fig. 7b and Additional file 5: Figure S5). This indicates that RA was able to partially compensate for loss-of-Efhc 2 function in the development of MCC. As compared with RA treated embryos, wild-type embryos treated with DEAB showed reduced MCC formation (Fig. 7c and Additional file 5: Figure S5). The expression of odf3 is seen adjacent to 2nd to 13 th somite in $48 \mathrm{hpf}$ embryos as compared to DMSO treated embryo, where its expression can be seen from 2nd to 15th somite (Fig. 7c). efhc2-Mo morphants treated with DEAB show almost or complete loss of odf3 expressing multi-ciliated cells (Fig. 7c and Additional file 5: Figure S5). Treatment of Efhc2-knock-down embryos with another RA inhibitor citral, also lead to similar reduction of number and domain of odf3 expressing MCC (Fig. 7d). This indicates that the inhibition of RA synthesis by
$\mathrm{DEAB}$ or citral leads to a dramatic loss of MCC formation in efhc2-Mo injected embryos. This could be interpreted as loss of Efhc 2 and RA signaling has a synergistic effect on reduced $\mathrm{MCC}$ formation. Taken together, our results suggest that RA and Efhc2 synergistically regulate MCC development (Fig. 8).

\section{Discussion}

Here, we have identified Efhc2 as a regulator of nephron segmentation in zebrafish. Knock-down of Efhc2 specifically affects the segmentation of distal pronephric tubule consisting of DE, CS, and DL. Development of the proximal tubule segments such as PCT and PST was largely unaffected in these embryos. The expression of efhc2 starts in a non-spatially restricted manner by $6 \mathrm{hpf}$. First localized expression of efhc 2 can be seen at $9 \mathrm{hpf}$ in kupffer's vesicle (KV). Kupffer's vesicle (KV) is a transiently present organ that contains cilia and has been shown to establish asymmetric cell signaling and gene expression [19]. In zebrafish, kupffer's vesicle (KV), pronephros, otic vesicle, olfactory placode and neuromast cells are the organs which have abundant cilia [20]. efhc2 expression in these organs indicates that Efhc2 may have a role in cilia development or function. Consistent with this hypothesis, we observe that Efhc2 is required for formation of the multi-ciliated cells (MCC) in the pronephros. It has been shown that DM10 domain-containing proteins in Chlamydomonas are bound to flagellar microtubules and are proposed to be involved in axonemal targeting and assembly [21]. Efhc2, which contains three DM10 domains [22], may have a similar role in assembly and stability of cilial axoneme [23].

The expression of efhc2 mRNA was not uniform in the PCT segment. The proximal part of the PCT had more intense expression of efhc2, whereas, the distal part had very low expression. Unlike zebrafish proximal tubule segments, which is divided into PCT and PST, the mammalian proximal tubule is divided into 3 segments (S1, S2 and S3) [10]. The Xenopus proximal segment is also thought to consist of 3 different segments (PT1, PT2 and PT3) [24]. It is not clear if the distal part of PCT lacking efhc2 expression represents

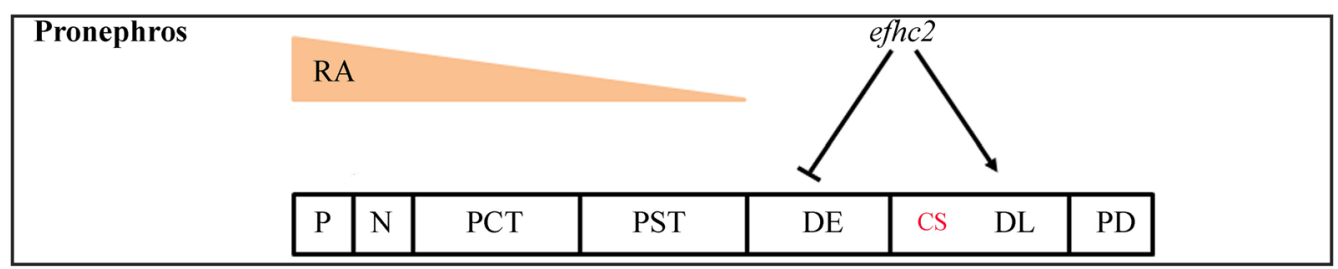

Fig. 8 Model of Efhc2 function during zebrafish pronephros development. RA signaling induces proximal and inhibits expansion of distal segments. Efhc2 is important for the segmentation of distal part of the pronephros. Efhc2 may act independently of RA signaling in the segmentation of pronephros 
a segment similar to PT2 and S2 seen in Xenopus and mammals respectively. efhc 2 expression could not be detected in pronephros in 72 hpf embryos, which could be due to low penetrance of the anti-sense probe at this stage. However, the expression of this gene in the neuromast cells was clearly visible. These mechanosensory cells are responsible for sensing water movement and contain cilia [25]. Knock-down of Efhc2 showed only mild morphological defects in zebrafish embryos. However, there was a significant impact of its loss-of-function on the segmentation of pronephros. Paired box 2 and 8 (Pax2a and Pax 8 ) act early during development in the intermediate mesoderm to specify pronephros. Hnf1ba/b act downstream of Pax $2 \mathrm{a} / 8$ to divide the pronephric region to rostral, central and caudal regions that give rise PCT, PST, DE and DL segments [13]. Hnf1ba/b transcription factors activate Irx3b in 10-15 somite zebrafish embryos that specify the PST and DE segments. However, in 15-28 somite embryos, Irx3b acts upstream of Hnf1ba/b to maintain its expression and specification of DE segment, whereas Hnf1ba alone is sufficient for maintaining DL segment [13]. RA signaling plays a major role is pronephros segmentation $[6,7]$. Our data suggests that Efhc2 acts independent of RA signaling in segmentation of distal nephric regions DE and DL. It would be interesting to see if Efhc2 acts downstream of transcription factors such as Hnf1ba/b. However, the effect of Efhc2 and RA signaling on MCC formation was synergistic. Hence, Efhc2 may have RA dependent and independent roles during zebrafish development.

Zebrafish $e f h c 1$ and $e f h c 2$ are paralogous genes with difference in the presence or absence of a C-terminal $\mathrm{Ca}^{2+}$ binding EF-hand domain. In zebrafish, Efhc1 does not have a $\mathrm{Ca}^{2+}$ binding EF-hand domain but Efhc 2 has this domain. Deletion studies of Xenopus Efhc1b show that, all three DM10 domains are expressed in the ciliary axonemes. The expression of Wnt8a is increased in absence of Efhc1b in Xenpous, indicating its role in regulating Wnt signaling pathway which is crucial for normal development [23]. In zebrafish, both $e f h c 2$ (in this study) and $e f h c 1$, are expressed in the pronephros. We show that zebrafish Efhc2 is required for segmentation of the distal part of pronephros. It will be of interest to identify the role of Efhc1 during zebrafish, particularly in pronephros development and function.

In summary, we have identified a novel component of genetic programme regulating pronephros segmentation in zebrafish. We find that Efhc2 regulates the distal segments of pronephros, the DE, CS, and DL. Efhc2 is expressed in ciliated cells and our work reveals a role for this gene in MCC development.

\section{Conclusions}

Our study has uncovered the role of efhc2 gene in the segmentation of distal part of the pronephros. We have also shown that RA and Efhc2 work independently in the process of pronephros segmentation, whereas they work synergistically in MCC development.

\section{Methods \\ Zebrafish husbandry}

Zebrafish wild-type strains Albino and TÜbingen (TÜ) were used in all experiments. The experiments carried out were approved by the institutional animal ethics committee. The embryos were staged according to Kimmel et al. [26].

\section{Zebrafish whole embryo in situ hybridization (WISH)}

Embryos at different stages [26] were fixed in 4\% PFA and stored in $100 \%$ methanol at $-20^{\circ} \mathrm{C}$. Whole-mount in situ hybridization was performed as previously described [27, 28]. Riboprobes against zebrafish efhc2, slc20a1a, trpm7, slc12a1, slc12a3, stc1, odf3 and $p d z k 1$ were synthesized and used. The DIG or Fluorescein labelled RNA probes were synthesized by linearizing the plasmids and transcribing with T7/SP6 RNA polymerases $(e f h c 2$, stcland $p d z k 1$ plasmids were linearized with $X h o I$ and transcribed with SP6, slc20a1a linearized with $\mathrm{XbaI}$ and transcribed with SP6, trpm7 linearized with SacII and transcribed with SP6, slc12a1 linearized with KpnI and transcribed with T7, slc12a3 and odf3 plasmids were linearized with $\mathrm{BamHI}$ and transcribed with T7. BM-purple and INT-BCIP (Roche) were used as chromogenic substrates to visualize the expression. Pictures were taken using Leica MZ16 stereo microscope.

\section{Morpholino and mRNA injection}

The antisense morpholino oligonucleotide was obtained from Gene Tools. The efhc2 splice-blocking morpholino (efhc2-Mo, 5' GTTTGATTCTGATGGTTCACCTTG $\mathrm{T}-3^{\prime}$ ) was designed targeting exon3/intron 3 splice donor junction and a mismatch morpholino (efhc2-MM) $5^{\prime}$ GTaTcATTCTcATGcTTCACCTTcT-3' with five base mismatches (small letters) was used as a control. The efhc2-ATG-Mo (5' TTCCAGGCAGCATTGGTAACG CCAT-3') was designed to block translation of efhc2 mRNA. Morpholino was dissolved in nuclease-free water as $1 \mathrm{mM}$ stock. 1 or $2 \mathrm{ng}$ of morpholino in $1 \mathrm{nl} 1 \mathrm{X}$ Danieau solution [ $58 \mathrm{mM} \mathrm{NaCl}, 0.7 \mathrm{mM} \mathrm{KCl}, 0.4 \mathrm{mM}$ $\mathrm{MgSO}_{4}, 0.6 \mathrm{mM} \mathrm{Ca}\left(\mathrm{NO}_{3}\right)_{2}, 5 \mathrm{mM}$ HEPES pH 7.6] was injected into 1-2 cell stage embryos using a Femtojet microinjector (Eppendorf).

For rescue experiments, full-length zebrafish efhc2 (2257 bp) was amplified by PCR. The forward primer 
contains EcoRI and the reverse primer contains XhoI sites. The efhc2-PCR amplicon was cloned into a pCRBluntII-Topo vector (Invitrogen) and was then subcloned into a pCS2 + vector at the EcoRI and XhoI sites. To synthesize efhc 2 mRNA, the efhc2-pCS2 vector was linearized with NotI and transcribed using SP6 mMessage mMachine kit (Ambion). To determine the optimal dose for rescue, 100,200 or 300 pg efhc 2 mRNA was coinjected with 2 ng efhc2-Mo morpholino. The optimal dose for rescue was found to be $200 \mathrm{pg}$.

\section{RT-PCR}

To check the efficiency of efhc2 splice-blocking morpholino, total RNA was isolated from embryos injected with efhc2-Mo and control embryos injected with efhc2MM. An equal amount of RNA was used to synthesize cDNA using superscriptIII kit (Invitrogen). The primers (flanking efhc2 exon 1 and exon 4) and PCR conditions were as follows: (forward $5^{\prime}$-CTGTCGTCCAACTGA GGGAAA-3', reverse $5^{\prime}$-CTCTTGTCGAAGGGTAGT ATAGGG- $3^{\prime}, \operatorname{Tm} 55{ }^{\circ} \mathrm{C}, 30$ cycles). Same PCR primers were used for amplifying efhc 2 from different developmental stages of zebrafish.

\section{Chemical treatment}

Retinoic acid (10 mM) and DEAB (1 M) (Sigma-Aldrich) were dissolved in $100 \%$ dimethyl sulfoxide (DMSO), aliquoted and stored at $-80{ }^{\circ} \mathrm{C}$. Citral was dissolved in $100 \%$ ethanol. Briefly, embryos were incubated in dark from 9 to $16 \mathrm{hpf}$ stage in $1 \times 10^{-7} \mathrm{M} \mathrm{RA} / \mathrm{DMSO}$, $1 \times 10^{-5} \mathrm{M} \mathrm{DEAB} / \mathrm{DMSO}$ and $2.5 \times 10^{-5} \mathrm{M} \mathrm{citral} /$ ethanol made with E3 embryo media. For control, embryos were incubated with either DMSO or ethanol. Embryos were fixed at 24 and $48 \mathrm{hpf}$ and analysed by WISH.

\section{Dextran injection}

To check the effect of Efhc2 knock-down on zebrafish pronephros function, we injected $40 \mathrm{kDa}$ fluorescein isothiocyanate dextran (Sigma) in common cardinal vein of $48 \mathrm{hpf}$ embryos [16]. Clearance of dextran was analysed at $72 \mathrm{hpf}$ and $96 \mathrm{hpf}$.

\section{Histology}

WISH stained embryos were fixed in 4\% PFA for $1-2 \mathrm{~h}$ at room temperature, washed with PBS and then transferred into 30\% sucrose in PBS for overnight. Next day, embryos were incubated in a 1:1 mixture of OCT and $30 \%$ sucrose/PBS for $30 \mathrm{~min}$. Embedding was done on plastic molds and polymerization of OCT was achieved by incubation on dry ice, samples were then stored at $-80^{\circ} \mathrm{C}$. Sectioning of embryos was done with the help of cryotome; $7-10 \mu \mathrm{m}$ thick cryo-sections were made and mounted on glass slides for microscopy.

\section{Additional files}

Additional file 1: Figure S1. efhc2-Mo inhibits pre-mRNA splicing. (A) Schematic representation of zebrafish efhc2 exon/intron organization, the target site of the splice-blocking efhc2 morpholino (efhc2-Mo) and the forward and reverse primers used in RT-PCR for amplification of efhc2. (B) CDNA was prepared from embryos injected with efhc2-Mo and efhc2-MM, PCR amplified, cloned into pCR Blunt II Topo vector (Invitrogen) and sequenced using SP6 and T7 primers. Sequencing shows that injection of efhc2-Mo leads to deletion of exon-2 and exon-3 of efhc2.

Additional file 2: Figure S2. Effect of Efhc2 knock-down on nephron segmentation. (A) Morphological defects seen by injection of efhc2-ATG-Mo. Arrow indicates mild pericardial oedema. (B) WISH showing expression of slc12al (DE), slc12a3 (DL), stC1 (CS) and odf3 (MCC) in efhc2-ATG-Mo injected and standard control injected embryos. (C) Summary of defects caused by Efhc2 translation blocking morpholino.

Additional file 3: Figure S3. Efhc2 knock-down results in nephron segmentation defects. (A) WISH for pronephros segment or MCC specific markers on 24 and $48 \mathrm{hpf}$ morpholino injected embryos. Expression of s/c20a1a (PCT), trpm7 (PST), s/c12a1 (DE), s/c12a3 (DL), stc1 (CS) and odf3 (MCC) in efhc2 mismatch control (efhc2-MM) and splice-blocking morpholino (efhc2-Mo) injected embryos. (B) Summary of defects caused by Efhc2 knock-down.

Additional file 4: Figure S4. Phenotype caused by over-expression of efhc2 mRNA. (A) efhc2 mRNA injected embryos showed dose-dependent pronephros defects.

Additional file 5: Figure S5. Role of RA and Efhc2 in pronephros segmentation. Wild-type embryos or efhc2-Mo morphants were treated with DMSO, RA and DEAB. WISH showing expression of s/c12al (DE), slc12a3 (DL), stc1 (CS) and odf3 (MCC). efhc2-Mo morphants treated with RA show expansion of expression domain of DE marker s/c12al and almost or complete loss of DL and CS markers s/c12a3 and stc1 as compared with wildtype embryos treated with RA. The expression domain and the number of cells expressing MCC maker odf3 was partially rescued by RA treatment in the morphants. DEAB treated efhc2-Mo morphants show expansion of $D E$, $\mathrm{DL}$, and CS as compared with wild-type embryos treated with DEAB. The expression domain of odf3 is reduced in DEAB treated WT embryos.

\section{Abbreviations}

OCT: optimal cutting temperature compound; PBS: phosphate-buffered saline; Hpf: hours post-fertilization.

\section{Authors' contributions}

PB carried out all experiments; PB, CP and RKS designed, analysed, interpreted the data and prepared the manuscript. All authors read and approved the final manuscript.

\section{Author details}

${ }^{1}$ Institute of Life Sciences, Nalco Square, Chandrasekharpur, Bhubaneswar, Odisha 751023, India. ${ }^{2}$ Manipal Academy of Higher Education, Manipal, Karnataka 576104, India. ${ }^{3}$ Agharkar Research Institute, Pune, Maharashtra, India. ${ }^{4}$ Savitribai Phule Pune University, Pune, Maharashtra, India.

\section{Acknowledgements}

This work was supported by a DBT-Ramalingaswami fellowship (BT/RLF/REENTRY/09/2010) and SERB-EMR (EMR/2016/003,780) to RKS. This work was also supported by intramural funds from ILS, which is an autonomous institute of DBT, Government of India. PB is a recipient of DBT-SRF. We thank Jaisen Mahankuda and Suryasikha Mohanty for technical help and Pranita Rout for maintaining zebrafish facility.

\section{Competing interests}

The authors declare that they have no competing interests.

\section{Ethics approval and consent to participate}

All animal experiments were approved by Institute Animal Ethics Committee (IAEC) of Institute of Life Sciences. 


\section{Publisher's Note}

Springer Nature remains neutral with regard to jurisdictional claims in published maps and institutional affiliations.

Received: 18 June 2018 Accepted: 5 October 2018

Published online: 16 October 2018

\section{References}

1. Takasato M, Little MH. The origin of the mammalian kidney: implications for recreating the kidney in vitro. Development. 2015;142(11):1937-47.

2. Costantini F, Kopan R. Patterning a complex organ: branching morphogenesis and nephron segmentation in kidney development. Dev Cell. 2010;18(5):698-712.

3. Dressler GR. The cellular basis of kidney development. Annu Rev Cell Dev Biol. 2006;22:509-29.

4. Drummond I. Making a zebrafish kidney: a tale of two tubes. Trends Cell Biol. 2003:13(7):357-65.

5. Drummond IA, Majumdar A, Hentschel H, Elger M, Solnica-Krezel L, Schier AF, Neuhauss SC, Stemple DL, Zwartkruis F, Rangini Z, et al. Early development of the zebrafish pronephros and analysis of mutations affecting pronephric function. Development. 1998;125(23):4655-67.

6. Wingert RA, Davidson AJ. The zebrafish pronephros: a model to study nephron segmentation. Kidney Int. 2008;73(10):1120-7.

7. Wingert RA, Selleck R, Yu J, Song HD, Chen Z, Song A, Zhou Y, Thisse B, Thisse C, McMahon AP, et al. The cdx genes and retinoic acid control the positioning and segmentation of the zebrafish pronephros. PLoS Genet. 2007;3(10):1922-38

8. Marra AN, Wingert RA. Roles of iroquois transcription factors in kidney development. Cell Dev Biol. 2014;3(1):1000131.

9. Pfeffer PL, Gerster T, Lun K, Brand M, Busslinger M. Characterization of three novel members of the zebrafish Pax2/5/8 family: dependency of Pax5 and Pax8 expression on the Pax2.1 (noi) function. Development. 1998;125(16):3063-74

10. Desgrange A, Cereghini S. Nephron patterning: lessons from xenopus, zebrafish, and mouse studies. Cells. 2015;4(3):483-99.

11. Bedell VM, Person AD, Larson JD, McLoon A, Balciunas D, Clark KJ, Neff KI Nelson KE, Bill BR, Schimmenti LA, et al. The lineage-specific gene ponzr1 is essential for zebrafish pronephric and pharyngeal arch development. Development. 2012;139(4):793-804.

12. Naylor RW, Davidson AJ. Hnf1 beta and nephron segmentation. Pediatr Nephrol. 2014:29(4):659-64.

13. Naylor RW, Przepiorski A, Ren Q, Yu J, Davidson AJ. HNF1 beta is essential for nephron segmentation during nephrogenesis. J Am Soc Nephrol JASN. 2013;24(1):77-87.
14. Cheng CN, Verdun VA, Wingert RA. Recent advances in elucidating the genetic mechanisms of nephrogenesis using zebrafish. Cells. 2015:4(2):218-33.

15. Gerlach GF, Wingert RA. Kidney organogenesis in the zebrafish: insights into vertebrate nephrogenesis and regeneration. Wiley interdiscip Rev Dev Biol. 2013;2(5):559-85.

16. Li Y, Cheng CN, Verdun VA, Wingert RA. Zebrafish nephrogenesis is regulated by interactions between retinoic acid, mecom, and Notch signaling. Dev Biol. 2014:386(1):111-22.

17. Cartry J, Nichane M, Ribes V, Colas A, Riou JF, Pieler T, Dolle P, Bellefroid EJ, Umbhauer M. Retinoic acid signaling is required for specification of pronephric cell fate. Dev Biol. 2006;299(1):35-51.

18. Liu Y, Pathak N, Kramer-Zucker A, Drummond IA. Notch signaling controls the differentiation of transporting epithelia and multiciliated cells in the zebrafish pronephros. Development. 2007;134(6):1111-22.

19. Kramer-Zucker AG, Olale F, Haycraft CJ, Yoder BK, Schier AF, Drummond IA. Cilia-driven fluid flow in the zebrafish pronephros, brain and Kupffer's vesicle is required for normal organogenesis. Development. 2005;132(8):1907-21.

20. Malicki J, Avanesov A, Li J, Yuan S, Sun Z. Analysis of cilia structure and function in zebrafish. Methods Cell Biol. 2011;101:39-74.

21. King SM. Axonemal protofilament ribbons, DM10 domains, and the link to juvenile myoclonic epilepsy. Cell Motil Cytoskelet. 2006;63(5):245-53.

22. Berrin T, Hikmet $Y$, Gulsen $V$, Ferda B, Erdal B, Ece $O$. No relation between EFHC2 gene polymorphism and Idiopathic generalized epilepsy. Afr Health Sci. 2015;15(4):1204-10.

23. Zhao Y, Shi J, Winey M, Klymkowsky MW. Identifying domains of EFHC1 involved in ciliary localization, ciliogenesis, and the regulation of Wnt signaling. Dev Biol. 2016;411(2):257-65.

24. Raciti D, Reggiani L, Geffers L, Jiang Q, Bacchion F, Subrizi AE, Clements D, Tindal C, Davidson DR, Kaissling B, et al. Organization of the pronephric kidney revealed by large-scale gene expression mapping. Genome Biol. 2008;9(5):R84.

25. Campeau E, Ruhl VE, Rodier F, Smith CL, Rahmberg BL, Fuss JO, Campisi J, Yaswen P, Cooper PK, Kaufman PD. A versatile viral system for expression and depletion of proteins in mammalian cells. PLoS ONE. 2009;4(8):e6529.

26. Kimmel CB, Ballard WW, Kimmel SR, Ullmann B, Schilling TF. Stages of embryonic development of the zebrafish. Dev Dyn. 1995;203(3):253-310.

27. Thisse C, Thisse B. High-resolution in situ hybridization to whole-mount zebrafish embryos. Nat Protoc. 2008;3(1):59-69.

28. Borah S, Barrodia P, Swain RK. Nucleolar protein 4-like has a complex expression pattern in zebrafish embryos. Int J Dev Biol. 2016;60(1-3):53-6.
Ready to submit your research? Choose BMC and benefit from:

- fast, convenient online submission

- thorough peer review by experienced researchers in your field

- rapid publication on acceptance

- support for research data, including large and complex data types

- gold Open Access which fosters wider collaboration and increased citations

- maximum visibility for your research: over 100M website views per year

At BMC, research is always in progress.

Learn more biomedcentral.com/submissions 